\title{
Magnetopause reconnection and interlinked flux tubes
}

\author{
F. R. Cardoso ${ }^{1,2}$, W. D. Gonzalez ${ }^{2}$, D. G. Sibeck ${ }^{3}$, M. Kuznetsova ${ }^{3}$, and D. Koga ${ }^{2}$ \\ ${ }^{1}$ EEL/USP - Escola de Engenharia de Lorena/Universidade de São Paulo, Lorena, São Paulo, Brazil \\ ${ }^{2}$ INPE - Instituto Nacional de Pesquisas Espaciais, São José dos Campos, São Paulo, Brazil \\ ${ }^{3}$ NASA Goddard Space Flight Center, Greenbelt, MD, USA \\ Correspondence to: F. R. Cardoso (flaviacardoso@usp.br)
}

Received: 30 April 2013 - Revised: 13 July 2013 - Accepted: 22 July 2013 - Published: 30 October 2013

\begin{abstract}
Magnetic reconnection can be a continuous or a transient process. Global magnetohydrodynamics (MHD) simulations are important tools to understand the relevant magnetic reconnection mechanisms and the resulting magnetic structures. We have studied magnetopause reconnection using a global 3-D MHD simulation in which the interplanetary magnetic field (IMF) has been set to large positive $B_{y}$ and large negative $B_{z}$ components, i.e., a south-duskward direction. Flux tubes have been observed even during these constant solar wind conditions. We have focused on the interlinked flux tubes event resulting from time-dependent, patchy and multiple reconnection. At the event onset, two reconnection modes seem to occur simultaneously: a timedependent, patchy and multiple reconnection for the subsolar region; and, a steady and large-scale reconnection for the regions far from the subsolar site.
\end{abstract}

Keywords. Magnetospheric physics (magnetopause, cusp, and boundary layers; solar wind-magnetosphere interactions)

\section{Introduction}

Magnetic reconnection has been considered a dominant mechanism for solar wind mass, momentum and energy transfer to the magnetosphere. This process can be distinguished as continuous (e.g., Gonzalez and Mozer, 1974) or transient (e.g., Russell and Elphic, 1978).

Time-varying and/or patchy reconnection may produce flux transfer events (FTEs). FTEs are mainly identified by bipolar $B_{\mathrm{n}}$ (component of the magnetic field normal to the magnetopause) signatures and enhanced core magnetic field (Russell and Elphic, 1978; Haerendel et al., 1978). The events contain a mixture of accelerated plasmas (Paschmann et al., 1982). FTEs typically have sizes on the order of $1 R_{\mathrm{E}}$ (Earth radii) in the direction normal to the magnetopause (Rijnbeek and Cowley, 1984).

The existent models do not agree on the azimuthal extent of an FTE (perpendicular direction to the motion of the FTE at the magnetopause plane). Lee and Fu (1985), Southwood et al. (1988) and Scholer (1988) proposed a larger extent of FTEs than Russell and Elphic (1978). The extent of an FTE is related to how this structure is generated in the magnetic reconnection site (Fear et al., 2008). Russell and Elphic (1978) proposed that an FTE is generated at a patchy reconnection region and its extent would be limited to this. Southwood et al. (1988) and Scholer (1988) proposed a model based on a single $X$ line which may extend for large azimuthal distances. Those two models (patchy and single $X$ line) produce pairs of FTEs. Lee and Fu (1985) suggested a model of multiple $X$ lines which have long extents similar to the single $X$ line model. In this case, the number of flux rope FTEs formed will be the number of $X$ lines minus 1 .

The FTE can be identified as a bundle of magnetic flux forming an "elbow" in the patchy reconnection model (Russell and Elphic, 1978). The flux tube is narrow in its azimuthal extent and has a circular cross-section of about $1 R_{\mathrm{E}}^{2}$. Flux tubes generated by the single and multiple $X$ line models may also have cross-sections of about $1 R_{\mathrm{E}}^{2}$, but long azimuthal extent along the magnetopause (Southwood et al., 1988; Scholer, 1988; Lee and Fu, 1985).

Even though the models do not agree on the FTE characteristics, they point out that magnetic reconnection would be the cause of the events. The magnetic reconnection process is likely to occur whenever the magnetic field lines can diffuse across the plasma under finite conductivity (or resistivity) (Treumann and Baumjohann, 1997). If the conductivity is high (negligible resistivity), the diffusion time can be very 
long and the magnetic field is not able to diffuse efficiently across the plasma. The resistivity must then be present in simulations to allow magnetic reconnection.

Magnetohydrodynamic (MHD) models can reproduce the effects of magnetic reconnection using physical or only numerical resistivity (numerical dissipation, numerical diffusion). Raeder (2006) studied the relation between numerical resistivity and grid resolution in MHD simulations. His results showed that the numerical resistivity is proportional to the square of the cell size. This indicates that the smaller the cell size the lower the numerical dissipation. A finer grid resolution is able to suppress the numerical diffusion in a manner that FTEs are allowed to form and grow (Raeder, 2006). FTEs are pointed out as a consequence of high grid resolution and low numerical dissipation in his simulations.

The early three-dimensional (3-D) MHD simulation of Ogino et al. (1989) showed a flux tube on the dayside magnetopause despite the low resolution used in their model. Fedder et al. (2002) also used MHD simulation and obtained the FTE described as a single magnetic structure instead of a pair. The simulated FTE propagated across one half of the dayside magnetopause presenting the observational characteristics of twisting of magnetic field lines and large core field. The structure was formed by unsteady magnetic reconnection at a single merging line.

Raeder (2006) associated the FTE generation with reconnection at multiple lines considering purely southward IMF (interplanetary magnetic field) and magnetic dipole tilt in his MHD simulation. He also applied small IMF $B_{y}$ on the system and obtained the same results as those for the southern IMF case. The only difference is that the FTE has an axial field component under $B_{y}$ influence and becomes a flux rope. The generation mechanism is proposed for new FTEs forming after one that already exists. The first FTE is formed as a result of the interaction between the solar wind with a southward IMF and Earth's dipolar magnetic field at the beginning of the simulation. There are two $X$ lines, one located at the flow stagnation point and the other one lower at the Southern Hemisphere. The first FTE moves southward. The two $X$ points also move with the FTE. The $X$ point located at the stagnation point stretches into two $Y$ points connected by a current sheet. The current sheet tears at the lower $Y$ point and becomes an $X$ point. The stagnation flowline impinges on the magnetopause and the northern $Y$ point turns into an $X$ point as well. At this point a new FTE is formed. The FTE grows and the cycle repeats. The dipole tilt causes all the dynamics to occur in the south.

Dorelli and Bhattacharjee (2009) pointed out that FTEs are produced by flow vortices and the multiple $X$ lines would be a consequence of these vortices. The process starts when the subsolar magnetopause current grows and changes the local geometry of the magnetic field, not the topology. The magnetic curvature forces the flow stagnation point away from the subsolar point. The large flow shear generates flow vortices that grow and coalesce, forming the FTE. The vortices within the FTE put in contact different magnetic topology regions and drive magnetic reconnection. Dorelli and Bhattacharjee (2009) included the $B_{y}$ component along with the southward IMF. Raeder (2006) obtained FTEs under purely southward IMF but considered earth magnetic dipole tilt. Southwood et al. (1988) believe that the internal magnetic field component along the FTE can be added by the influence of either a magnetic field shear or a flow shear (Fear et al., 2008).

$\mathrm{Ku}$ and Sibeck (1998) investigated FTEs forming by bursty merging at the magnetopause along an extended $X$ line through two-dimensional MHD simulation. They did not find the typical symmetric bipolar signatures in the magnetic field normal to the magnetopause. Instead, the signatures were of asymmetric bipolar type. Their conclusion was that a single $X$ line did not seem to be the dominant mechanism for the production of transient events at the dayside magnetopause.

$\mathrm{Ku}$ and Sibeck (2000) also worked on FTEs developing from multiple $X$ lines. They obtained bulges followed by islands. The bulges are characterized by asymmetric bipolar signatures normal to the magnetopause while islands show symmetric features. The bulges were confined to the magnetosheath and the islands generated significant signatures in the magnetosphere. The bipolar signatures were followed by transitions from the magnetosheath to the magnetosphere. They claim that crater FTEs with a strong core field should be common.

Sibeck et al. (2008) modeled a crater FTE detected in Themis observations using an MHD simulation. A spacecraft entering a crater FTE should observe magnetic field strength enhancements bounding trench-like magnetic field strength decreases, which in turn bound a strong core magnetic field (Sibeck et al., 2008). This feature may occur in the absence of guide fields. Kuznetsova et al. (2009) worked on a different event from the same run as Sibeck et al. (2008) but focusing on the FTE wake region of weak magnetosheath magnetic field strengths.

Hesse et al. (1990) found a peculiar FTE shape of two elbowlike interlinked flux tubes for large shear angles. They proposed a magnetic field model in which localized perturbations are introduced at the magnetopause. The "elbow" topology is similar to the model of Russell and Elphic (1978) although their tubes are not interconnected. Another difference is that the flux tubes have no internal twist in the Hesse et al. model. Louarn et al. (2004) observed the interlinked tubes in Cluster data. They claim that patchy reconnection occurring at multiple points would explain the observations. Otto (1995) produced the interlinked tubes in local MHD simulations by enhancing resistivity at certain sites. The flux tubes are generated as a result of reconnection at multiple patches in their simulation. Lee et al. (1993) attributed the formation of the interlinked tubes to a combination of multiple $X$ lines and patchy reconnection. 
Lee et al. (1993) claim that the evolution of the interlinked flux tubes would be a consequence of further reconnection, leading to one tube connected only to the geomagnetic field and the other tube only to the IMF. This characteristic may not be observed in a MHD simulation using localized sites of enhanced resistivity (Lee et al., 1993). Nishida (1989) pointed out that the formation of the low-latitude boundary layer (LLBL) would be a consequence of the reconnection between the interlinked flux tubes.

Otto (1995) investigated the evolution of the interlinked tubes in 3-D MHD simulation for two cases: (1) not allowing reconnection between them at the interface of the flux tubes through ideal MHD and (2) allowing reconnection introducing resistivity at the interface. Their results show that a singular current sheet strongly tilted compared to the original sheet is formed at the interface if no resistivity is present. In the presence of resistivity the interaction between the tubes generates helically twisted closed magnetospheric flux.

In this paper we investigate the formation of FTEs and magnetic reconnection at the subsolar magnetopause using a global 3-D MHD simulation under a large amplitude southduskward IMF. We will examine the results from only one very high resolution run. We focus on a large-scale interlinked flux tubes event. We describe the reconnection modes present in the simulation and during the interlinked tubes event. This work provides the global perpective of the largescale structure of the FTE. The achievement of this picture is a difficult task even with the recent multi-spacecraft missions. Our present work is as an attempt at collaborating with the observations.

The simulation setup and description is displayed in Sect. 2. Our results are discussed in Sect. 3. We focus on the interlinked flux tubes event in Sect. 3.1 and show a glance after the event in Sect. 3.2. Last, we point out our final conclusions in Sect. 4.

\section{Simulation description}

We have worked on a 3-D MHD simulation processed at the Community Coordinated Modeling Center (CCMC). The global MHD code used was BATS-R-US (BlockAdaptive-Tree-Solarwind-Roe-Upwind-Scheme), developed by the Center for Space Environment Modeling at the University of Michigan (Powell et al., 1999; Ridley et al., 2004; Toth et al., 2005). The code solves the ideal MHD equations (conservation of mass, momentum and energy equations, and the Faraday's law) with a finite volume discretization (Kuznetsova et al., 2007). The code solves the equations using numerical methods related to Roe's Approximate Riemann Solver. The details about the full set of equations and the numerical method can be found in Powell et al. (1999) and Gombosi et al. (2004).

The simulation grid is composed of many self-similar three-dimensional rectangular blocks arranged in varying degrees of spatial refinement levels (Kuznetsova et al., 2007). The near magnetopause boundary layer region is highly resolved, presenting a grid resolution of $1 / 16 R_{\mathrm{E}}$. Figure 1 shows the plasma pressure for the $x-z$ plane at $y=0$ at the beginning of the simulation. At this time the magnetopause subsolar point is located at $x=8.0$ and $z=0 R_{\mathrm{E}}$. The finer resolution can be noticed around the magnetopause boundary layer. Such high resolution permits tracking flux tubes and FTEs, as pointed out by Raeder (2006).

The inner boundary of the magnetosphere is set at $3 R_{\mathrm{E}}$. The magnetospheric MHD part is attached to an ionospheric potential solver named Ionosphere Electrodynamics (IE) (Toth et al., 2005). This module uses magnetospheric fieldaligned currents to generate an auroral precipitation pattern. The auroral pattern and the solar illumination are used to calculate Hall and Pedersen conductances (Robinson et al., 1987; Moen and Brekke, 1993). The IE provides electric potentials to the magnetospheric MHD module.

The magnetic reconnection occurs due to the numerical dissipation (numerical resistivity). The dissipation regions are very localized at the discontinuities as the bow shock and the magnetopause (Fedder et al., 2002). Dorelli and Bhattacharjee (2009) pointed out that the level of numerical resistivity is significantly smaller in Raeder's (2006) than in Fedder et al. (2002) simulation due the finer grid resolution of $0.08 R_{\mathrm{E}}$. In our simulation the grid resolution is $1 / 16=0.06 R_{\mathrm{E}}$ and the level of numerical resistivity may be still smaller than in Raeder's simulation. Fedder et al. (2002) pointed out that the magnetic merging rate between the IMF and the geomagnetic field is controlled by the solar wind parameters and by the ionospheric conductance, not by the resistivity. However, even if the overall merging rate does not depend on numerical resistivity, the physical structure of the solution and possible instabilities do depend on it.

The input initial conditions have been set at $5.0 \mathrm{ncc}^{-1}$ for the solar wind density and $600 \mathrm{~km} \mathrm{~s}^{-1}$ for the $X$ component of the velocity. The IMF clock angle has been set at 135 degrees (south-duskward IMF), in which the IMF $y$ and $z$ components are $B_{y}=+14 \mathrm{nT}$ and $B_{z}=-14 \mathrm{nT}$. There is no dipole tilt. The IMF is imposed all over the simulation box at the beginning of the simulation.

On a global perspective of our simulation, the magnetopause lies about $8.5 R_{\mathrm{E}}$ from Earth along the Sun-Earth line. We observed several FTE-like structures during $45 \mathrm{~min}$ of simulation. Although the solar wind conditions are kept constant during the simulation, FTEs were generated, indicating that local instabilities during constant solar wind conditions can trigger time-dependent magnetic reconnection (Dorelli and Bhattacharjee, 2009). Raeder (2006) emphasizes that the constant input conditions show the spontaneous generation of the FTEs without any identified forcing agent.

The simulation has been divided in three stages in order to make our comprehension easier. In the first stage reconnection is occurring along an extended $X$ line. Secondly, a 


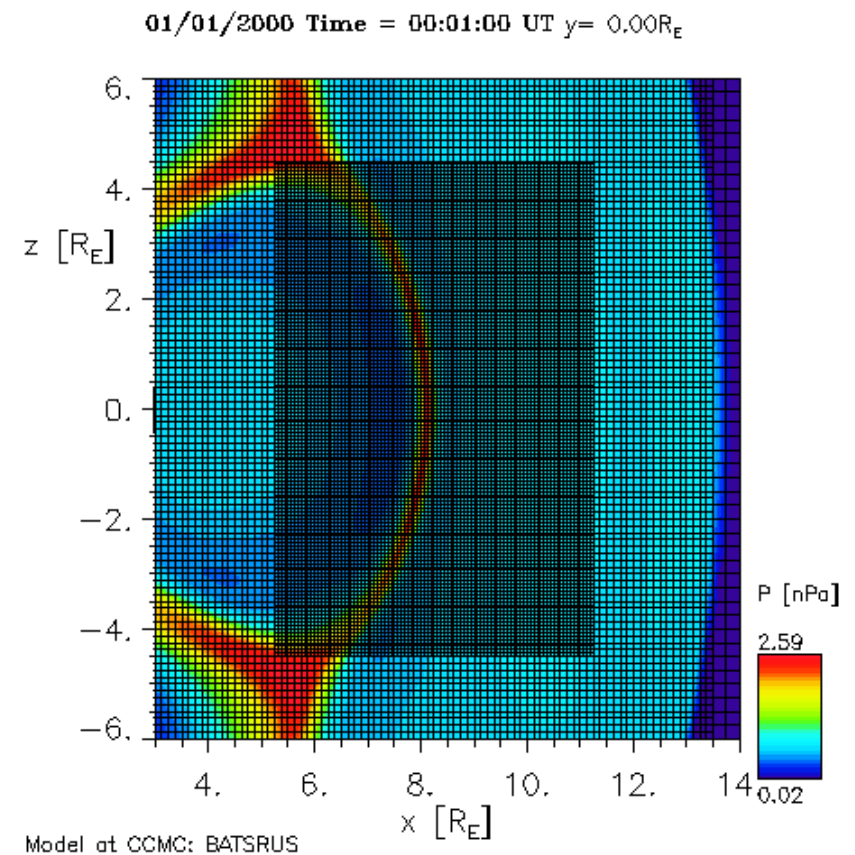

Fig. 1. Plasma pressure for the $x-z$ plane at $y=0$ at the beginning of the simulation. Earth is located at $(0,0,0)$. The finer resolution of $1 / 16 R_{\mathrm{E}}$ is located around the magnetopause boundary layer. At this time the magnetopause subsolar point is located at $x=8 R_{\mathrm{E}}$ and $z=0$.

large and intense pressure bulge appears at the subsolar region simultaneously with a magnetic topology change, which later moves northward. This structure is of the interlinked flux tubes type. During the third stage, other structures have been seen at the magnetopause region, but shifted from the subsolar point and moving randomly poleward.

\section{Results}

During the first stage of the simulation, which takes about $23 \mathrm{~min}$, reconnection seems to be occurring along an extended $X$ line. The tilted dashed line in the sketch in Fig. 2 indicates the $X$ line on the magnetopause for the $y-z$ plane and the arrows represent the flow leaving the reconnection line. The lines at $z=+1,+0.5,0,-0.5$, and $-1 R_{\mathrm{E}}$ in Fig. 2 refer to the plots in Fig. 3. The $z$ cuts (Fig. 3) show the $z$ component of the velocity for the $x-y$ planes. The blue and red regions roughly correspond to the flow propagating southward and northward, respectively. The flow reversal regions draw a line that separates the northward (red) and southward (blue) flows. For $z=0$, the flow reversal region is located near the subsolar point $(8.45,0,0)$, while for positive $z$ plots it is shifted to the dusk side and for negative $z$ plots to the dawn side. The tilted $X$ line separates the oppositely directed flows on the magnetopause. The line is oriented from

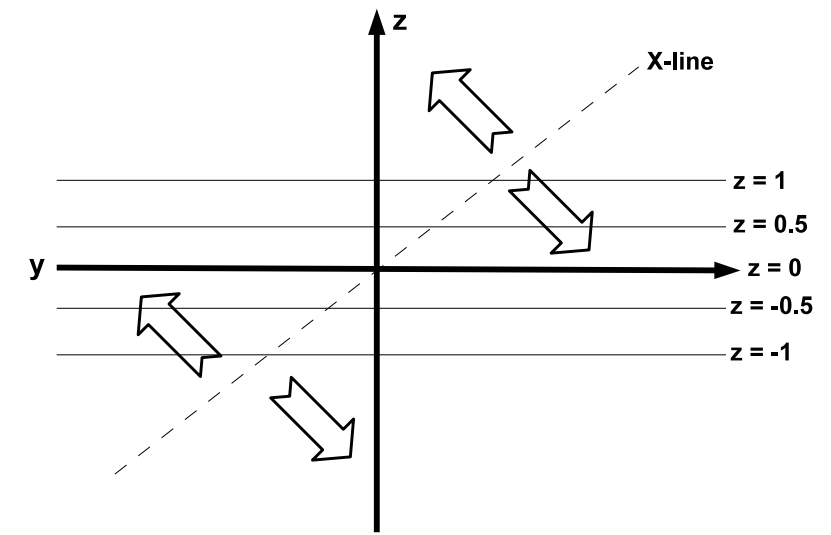

Fig. 2. Sketch of the magnetopause for the $y-z$ plane. The tilted dashed line indicates the $X$ line. The arrows represent the flow leaving the reconnection line. The lines at $z=+1,+0.5,0,-0.5$, and $-1 R_{\mathrm{E}}$ indicate the $z$ cuts shown in Fig. 3 .

southern dawn to northern dusk, in agreement with Gonzalez and Mozer (1974) for the southward and duskward IMF.

During the second phase of the simulation, a large magnetic structure appears at the subsolar magnetopause. The peculiar configuration of this FTE corresponds to the two interlinked elbow-shaped flux tubes (Hesse et al., 1990; Lee et al., 1993; Otto, 1995; Louarn et al., 2004). We have investigated more carefully this interesting magnetic structure and the results will be discussed in Sect. 3.1. After roughly four minutes growing at the subsolar region, the interlinked flux tubes move northward. They move away from the reconnection site due to the magnetic tension and the magnetosheath flow (Lee et al., 1993; Fear et al., 2008).

The third phase starts after the formation and propagation of the interlinked flux tube structure. Several FTE-like structures appear at regions shifted from the subsolar point and move randomly poleward, to the north or south. We have investigated the structures right after the interlinked flux tube event. The discussion is shown in Sect. 3.2.

\subsection{Interlinked flux tubes}

We have analyzed the large-scale magnetic structure of the two interlinked flux tubes at the subsolar magnetopause. The two interconnected tubes are illustrated in our sketch in Fig. 4b. The magnetic field lines and the two tubes are displayed on the magnetopause as seen from the Sun. The Xs indicate the possible configuration of the two reconnection sites responsible for this structure formation. The arrows indicate that the reconnected magnetic field lines flow northward and southward. The two interconnected tubes have one side connected to Earth and the other to the IMF. At the subsolar region the tube on the top is connected to the Southern Hemisphere and the tube on the bottom to the northern side. These tubes are forced to move against each other due to the 

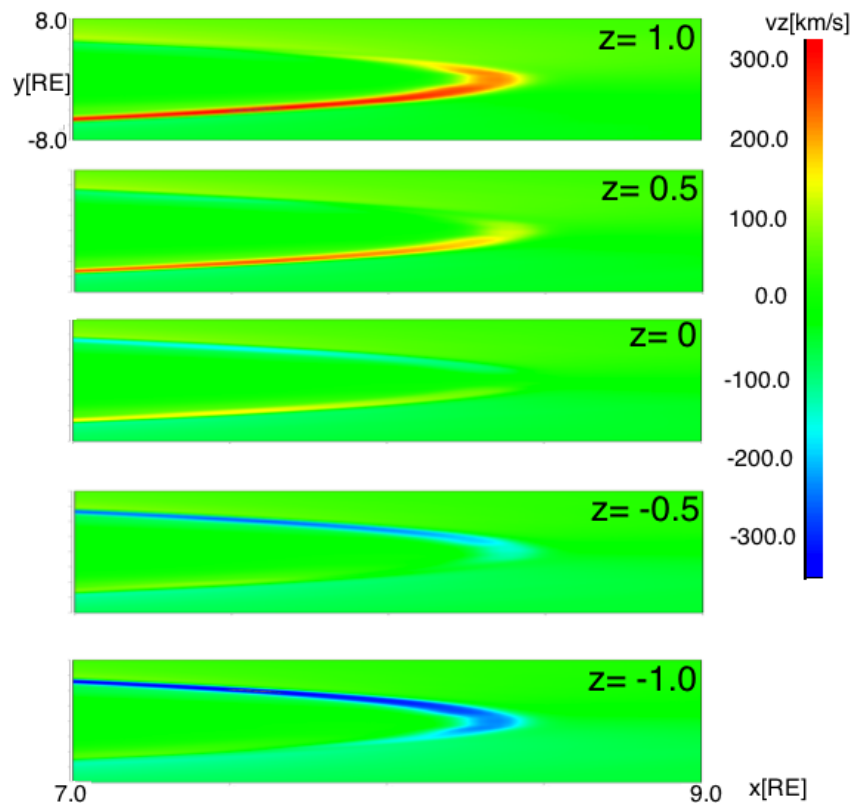

Fig. 3. Velocity in the $z$ direction for $z$ cuts $(z=+1,+0.5,0,-0.5$, $-1 R_{\mathrm{E}}$ ) on the $x-y$ plane. The blue (red) region shows the flow direction roughly to south (north). The $X$ line is located at the point of flow reversal.

magnetic stress resulting from magnetic field gradient and curvature.

We checked if the FTE signatures discussed in Sect. 1 are present in the interlinked flux tubes. First we analyzed each flux tube independently. Figures 5 and 6 show the magnetic field data $\left(B_{x}, B_{z}, B\right)$ from two virtual satellites crossing the northern $(z=0.2)$ and the southern $(z=-0.2)$ part of the interlinked flux tubes, respectively. The data is displayed as follows: the red line refers to $B$, the black to $B_{x}$ and the blue to $B_{z}$. The satellites move from the magnetosphere $\left(x=8.2 R_{\mathrm{E}}\right)$ to the magnetosheath $\left(x=8.6 R_{\mathrm{E}}\right)$ at $y=0$. The satellite trajectories comprise the time before the interlinked flux tubes, and during their formation and propagation. The data time series start in the magnetosphere at $t=00: 23: 00,1 \mathrm{~min}$ before the interlinked flux tubes onset. The trajectory ends in the magnetosheath at $t=00: 28: 00$, $4 \mathrm{~min}$ after the tubes onset. The tubes start moving northward after $t=00: 27: 00$.

As the tubes are located near the subsolar point, the normal component of the magnetic field $B_{\mathrm{n}}$ can be approximated by $B_{x}$. In both figures, $B_{x}$ is about zero in the magnetosphere. While the satellite moves towards the magnetosheath, $B_{x}$ (black line) increases positively on the northern side (Fig. 5). For the Southern Hemisphere (Fig. 6), $B_{x}$ becomes more negative. We have not observed the typical bipolar $B_{\mathrm{n}}$ signatures (Russell and Elphic, 1978; Haerendel et al., 1978) in each flux tube independently. That indicates that the tubes do not exhibit twisting of the magnetic field

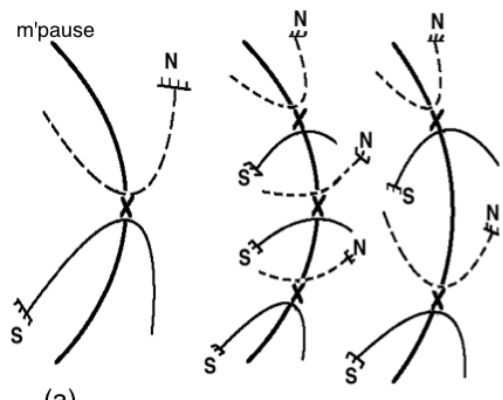

(a)

(b)

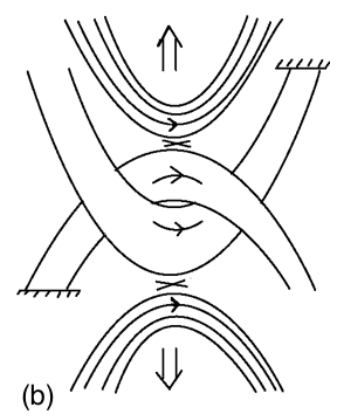

Fig. 4. (a) Sketch of the three steps in the $X$ point configurations for the magnetic topology evolution in Fig. 9. The left panel in the sketch is the configuration for the topology shown at $t=-30$ and $-15 \mathrm{~s}$ (first two panels in Fig. 9), the middle sketch refers to $t=0$ (middle panel) and the sketch on the right to $t=15$ and $120 \mathrm{~s}$ (two panels on the right). The m'pause line refers to the magnetopause and the Xs to the $X$ points. The full and dashed lines are the reconnected magnetic field lines coming out from the reconnection site, and connected to Earth's Southern (S) and Northern (N) Hemispheres. (b) Sketch illustrating the structure of two interlinked flux tubes. We show magnetic field lines and tubes on the magnetopause seen from the Sun. The Xs indicate the two possible reconnection sites responsible for such magnetic field configuration.

lines, in agreement with the interlinked flux tubes obtained by Hesse et al. (1990).

The magnetic field magnitude $B$ (red line) presents a decrease in both Figs. 5 and 6 at about 00:24:40 (hh:mm:ss). At that time the satellites get closer to the tubes in the magnetosphere side but not inside them yet. The decrease of $B$ is probably due to the entry into the current layer, as explained by Sibeck et al. (2008). The satellites reach the tubes at about 00:25:30 and do not leave the structure. The tubes start growing into the magnetosheath at roughly 00:26:15. The system becomes anti-symmetric in the $x$ direction. The magnitude $B$ reaches a maximum at 00:25:45 and decreases again when the tubes start growing. The small enhancement of the magnitude $B$ inside the tubes could be related to the core enhancement described in FTEs (Russell and Elphic, 1978; Haerendel et al., 1978). The tubes reach the maximum distance in the $x$ direction into the magnetosheath (at $x=8.73 R_{\mathrm{E}}$ ) at 00:27:40. The magnitude $B$ increases in the northern side while it decreases in the Southern Hemisphere (Figs. 5 and 6) after 00:27:00, coincident with the tubes start propagating northward. $B_{z}$ (blue line) decreases from the magnetosphere to the magnetosheath for both hemispheres, as we expect.

As each flux tube did not present clear FTE signatures independently, we analyzed the two tubes as a unique FTE. Figure 7 shows the magnetic field data $\left(B_{x}, B_{z}, B\right)$ from a virtual satellite crossing the interlinked flux tubes in the $z$ direction. The satellite goes from $z=-1$ to $1 R_{\mathrm{E}}$ at $x=8.5 R_{\mathrm{E}}$ and $y=0 . B_{x}$ (black line) shows the symmetric bipolar characteristic. The magnetic field magnitude $B$ (red line) presents 


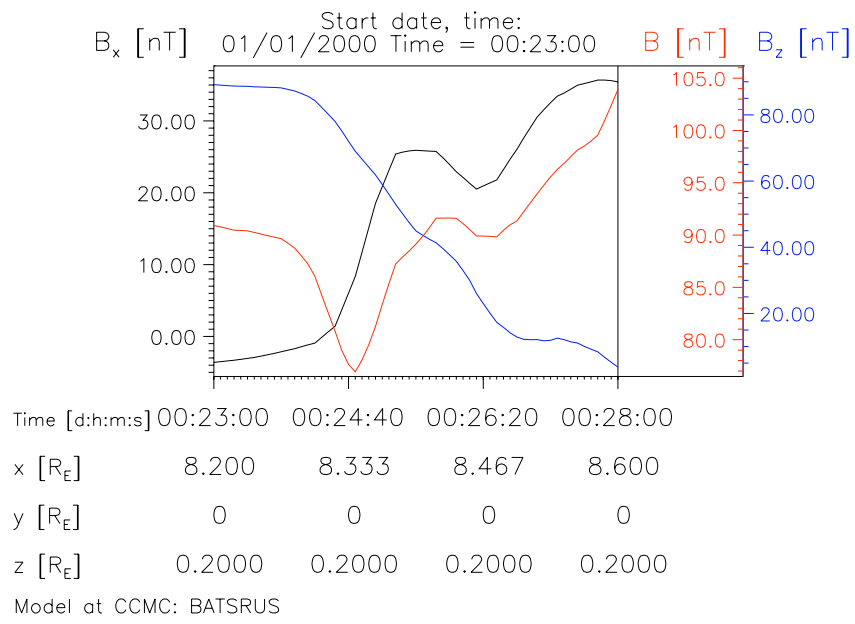

Fig. 5. Magnetic field data $\left(B_{x}, B_{z}, B\right)$ from a virtual satellite crossing the northern part of the interlinked flux tubes. The satellite trajectory goes from the magnetosphere $\left(x=8.2 R_{\mathrm{E}}\right)$ to the magnetosheath $\left(x=8.6 R_{E}\right)$ at $y=0$ and $z=0.2 R_{\mathrm{E}}$. Red line refers to $B$, black to $B_{x}$ and blue to $B_{z}$.

the core enhancement. These two features identify the set of the two interlinked flux tubes as an FTE.

We will corroborate the idea of the two interlinked flux tubes forming this peculiar FTE (Fig. 4b) throughout this section. We show the magnetic topology time evolution of the flux tubes formation from two perspectives: a cut at the frontside magnetopause ( $y-z$ plane at $x=8.41 R_{\mathrm{E}}$ ) looking anti-sunward in Fig. 8, and a cut at the noon-midnight plane $(x-z$ plane at $y=0)$ in Fig. 9. The snapshots have been taken at the time $t=-30,-15,0$ and $+15 \mathrm{~s}$. The time $t=120 \mathrm{~s}$ is shown only in Fig. 9. The magnetic topology changes at $t=$ 0 on the subsolar magnetopause. This time has been considered the onset of the interlinked flux tubes event. The onset time $t=0$ corresponds to the simulation time of $t=24 \mathrm{~min}$. The other snapshot times $(t=-30,-15,+15 \mathrm{~s},+120 \mathrm{~s})$ are counted from $t=0$. The colors refer to the magnetic field line topologies: red, closed; yellow, connected to north; green, connected to south; and blue, solar wind IMF. Hereinafter, all the magnetic topology plots will follow this color legend. The arrows refer to the plasma velocity and show the flow diverging from the reconnection region.

The configuration of the possible $X$ points for the magnetic topology evolution (Figs. 8 and 9) is shown in our sketch in Fig. 4a. The sketch presents the three steps of the $X$ point configuration at the noon-midnight cut. The left panel in Fig. 4a indicates the possible configuration for the topology shown at $t=-30$ and $-15 \mathrm{~s}$, the first two panels in Fig. 9. The middle sketch refers to $t=0$ at the middle panel in Fig. 9. The sketch on the right represents $t=15$ and $120 \mathrm{~s}$ at the two panels on the right in Fig. 9. The m'pause line refers to the magnetopause boundary layer and the Xs to the $X$ points. The full and dashed curved lines are the

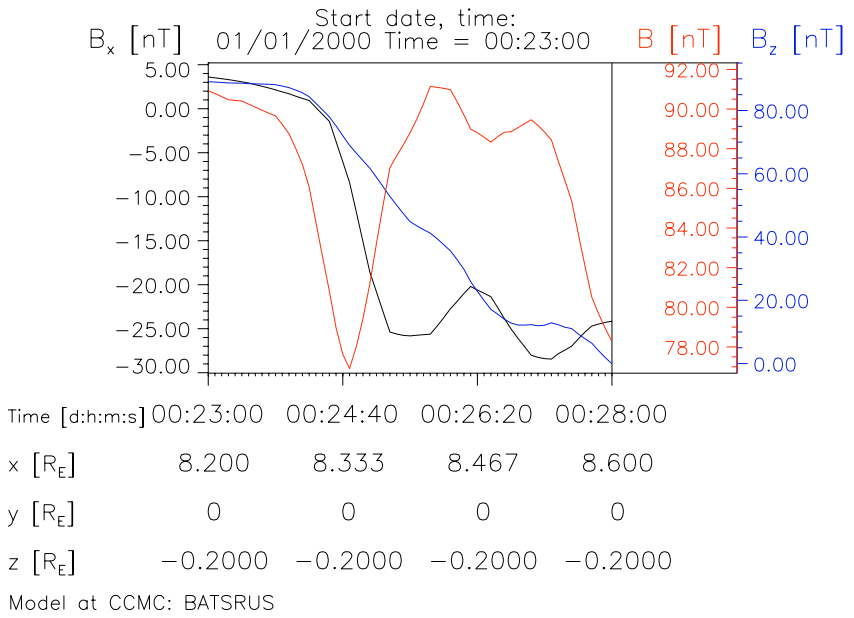

Fig. 6. Magnetic field data $\left(B_{x}, B_{z}, B\right)$ from a virtual satellite crossing the southern part of the interlinked flux tubes. The satellite trajectory goes from the magnetosphere $\left(x=8.2 R_{\mathrm{E}}\right)$ to the magnetosheath $\left(x=8.6 R_{\mathrm{E}}\right)$ at $y=0$ and $z=-0.2 R_{\mathrm{E}}$.
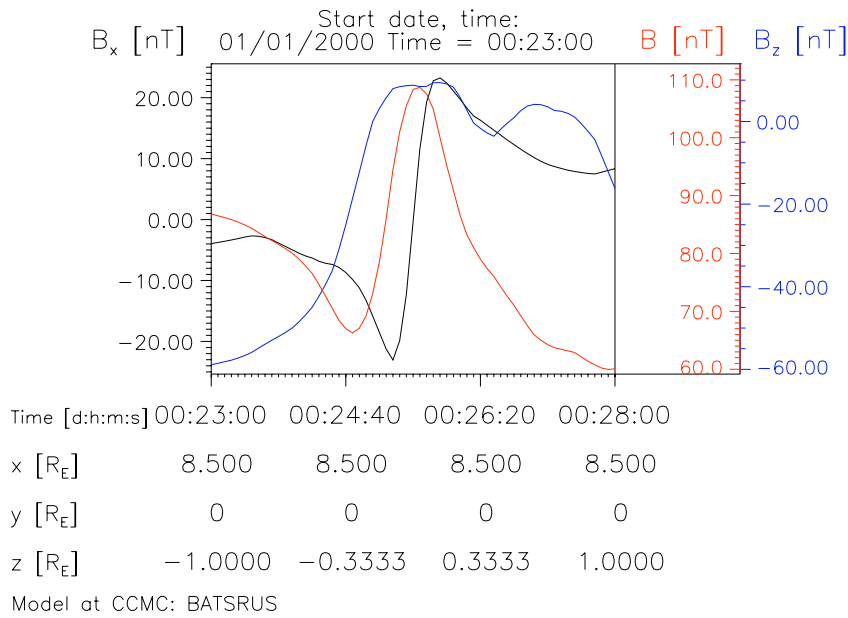

Fig. 7. Magnetic field data $\left(B_{x}, B_{z}, B\right)$ from a virtual satellite crossing the interlinked flux tubes in the $z$ direction. The satellite trajectory goes from $z=-1$ to $1 R_{\mathrm{E}}$ at $x=8.5 R_{\mathrm{E}}$ and $y=0$.

reconnected magnetic field lines coming out from the $X$ points and connected to Earth's Southern (S) and Northern (N) Hemispheres, respectively.

The left panel in Fig. 4a illustrates the single $X$ point seen at $t=-30$ and $-15 \mathrm{~s}$ in Figs. 8 and 9. The $X$ point location appears to be where the four topology colors meet in Fig. 9. The magnetic field line topology at $t=-30 \mathrm{~s}$ suggests that reconnection is occurring along an extended $X$ line. The $X$ line is indicated by a black trace in Fig. 8. We observed a perturbation on the semi-open magnetic field line regions (yellow and green) at $t=-15 \mathrm{~s}$ (Figs. 8 and 9) on the north dawn side and on the south dusk side, preceding the interlinked tubes onset. 

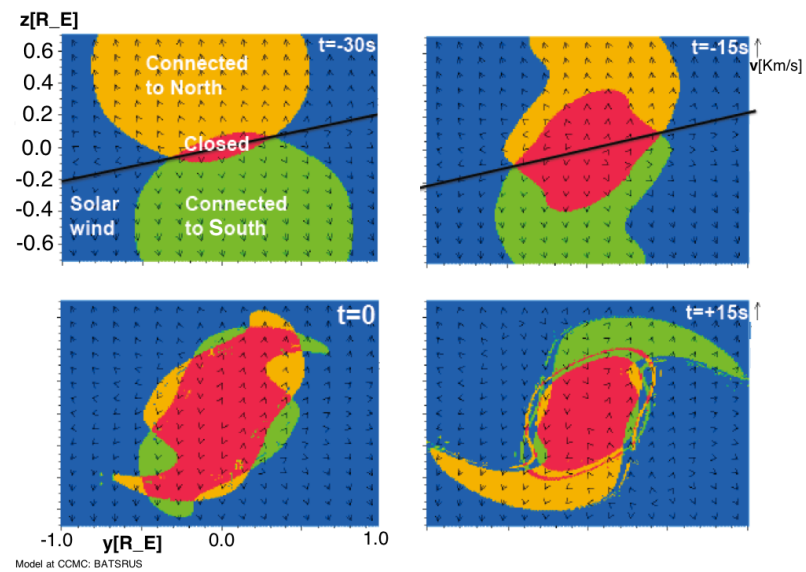

Fig. 8. Magnetic topology for the $y-z$ plane at $x=8.41 R_{\mathrm{E}}$, looking anti-sunward, for different time instants: $t=-30,-15,0,+15 \mathrm{~s}$, considering $t=0$ as the time when the magnetic topology changes (interlinked flux tubes onset). Colors are related to the magnetic topology as red, closed; yellow, connected to north; green, connected to south; and blue, solar wind. Arrows indicate flow velocity. The black line indicates the single and tilted $X$ line before the interlinked flux tubes onset.

The magnetic topology changed and became more complex at $t=0$, as shown in Figs. 8 and 9. Four blobs appeared at the magnetopause in Fig. 9. The yellow blobs are formed by the reconnected lines linked to the Northern Hemisphere and the green blobs to the Southern. The blobs correspond to magnetic flux tubes. The sketch of the these tubes is shown in the middle panel of Fig. 4a. We indicated the possible configuration of the reconnection sites that matches the magnetic topology seen in the middle panel of Fig. 9. The dashed lines connected to the Northern Hemisphere represent the yellow blobs. The full lines connected to the southern side refer to the green blobs.

We believe that in this case the $X$ points are not simply located where the four topologies meet, as pointed out by Dorelli and Bhattacharjee (2009). Instead, we analyzed the 3-D picture of the tubes and their connection to Earth. The snapshot at $t=0$ in Fig. 8 shows a cut of the four tubes on the magnetopause plane. Each tube appears as a pair of blobs. The four blobs seen at $t=0$ in Fig. 9 split into eight blobs in the perspective shown at $t=0$ in Fig. 8. However, there is a total of ten blobs in Fig. 8. The two missing blobs on this counting correspond to the tubes on the top and on the bottom of the middle sketch in Fig. 4a. In this case each tube corresponds to only one blob, and not to a pair. Based on our 3 -D analysis we proposed that two new $X$ points appeared in the north and south sides at $t=0$ (middle sketch in Fig. 4a), making a total of three $X$ points. The magnetic configuration changed from a single $X$ line (left sketch in Fig. 4a) to multiple reconnection lines (middle sketch in Fig. 4a). Our results agree with Lee and Fu (1985) who suggested that FTEs are generated by multiple $X$ lines.
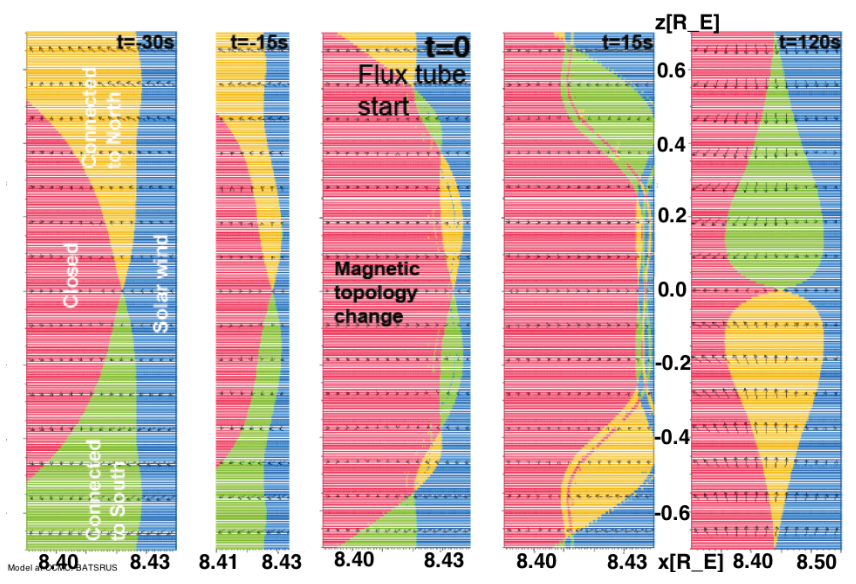

Fig. 9. Magnetic topology for the noon-midnight plane ( $x-z$ plane) for different time instants: $t=-30,-15,0,+15,120 \mathrm{~s}$, considering $t=0$ as the time when the magnetic topology changes (interlinked flux tubes onset). Colors are related to the magnetic topology as red, closed; yellow, connected to north; green, connected to south; and blue, solar wind. Arrows indicate flow velocity.

As time evolves the green and yellow bubbles near the subsolar region at $t=+15 \mathrm{~s}$ (Figs. 8 and 9) decrease in size. This decrease implies that the reconnection process occurring at the subsolar point is somehow suppressed. The $X$ point located at the subsolar site (middle sketch of Fig. 4a) seems to disappear. This means that the dashed line connected to the Northern Hemisphere at the near top side (yellow blob) and the full line at the near bottom side (green blob) of the subsolar $X$ point stopped being generated. Only the more recently formed two $X$ points remained at the magnetopause, as shown in the last panel in Fig. 4a. The two bubbles located further from the subsolar point increased while the two bubbles around the subsolar point vanished. The complicated magnetic topology seen at $t=0$ (Figs. 8 and 9) evolved into a simpler structure, as observed at $t=+120 \mathrm{~s}$ in Fig. 9. This structure corresponds to the two interconnected tubes shown in Fig. 4b. The tube on the top (large green blob) is connected to the Southern Hemisphere and the tube on the bottom (large yellow blob) to the northern side.

The plasma pressure evolution is shown in Fig. 10 at $t=0$ (top image), the event onset, and at $t=60 \mathrm{~s}$ (bottom image). The $z$ cuts $\left(z=0,1,2,3,4 R_{\mathrm{E}}\right)$ on the Northern Hemisphere are seen from the perspective of an observer viewing from below. Stars mark bulges of intense pressure at $t=60 \mathrm{~s}$. The black line connecting the stars suggest that the structure has the configuration of an elbow-shaped flux tube, as proposed by Russell and Elphic (1978). The event bulges outward into the magnetosheath at $y<0$ (dawnward, left side), but inward into the magnetosphere near $y=0$. We observed a similar pattern in the Southern Hemisphere. Therefore, we identified one elbow-shaped tube in the Northern Hemisphere $(t=60 \mathrm{~s}$ in Fig. 10) and another one in the Southern Hemisphere. The event in the southern bulges outward into the magnetosheath 

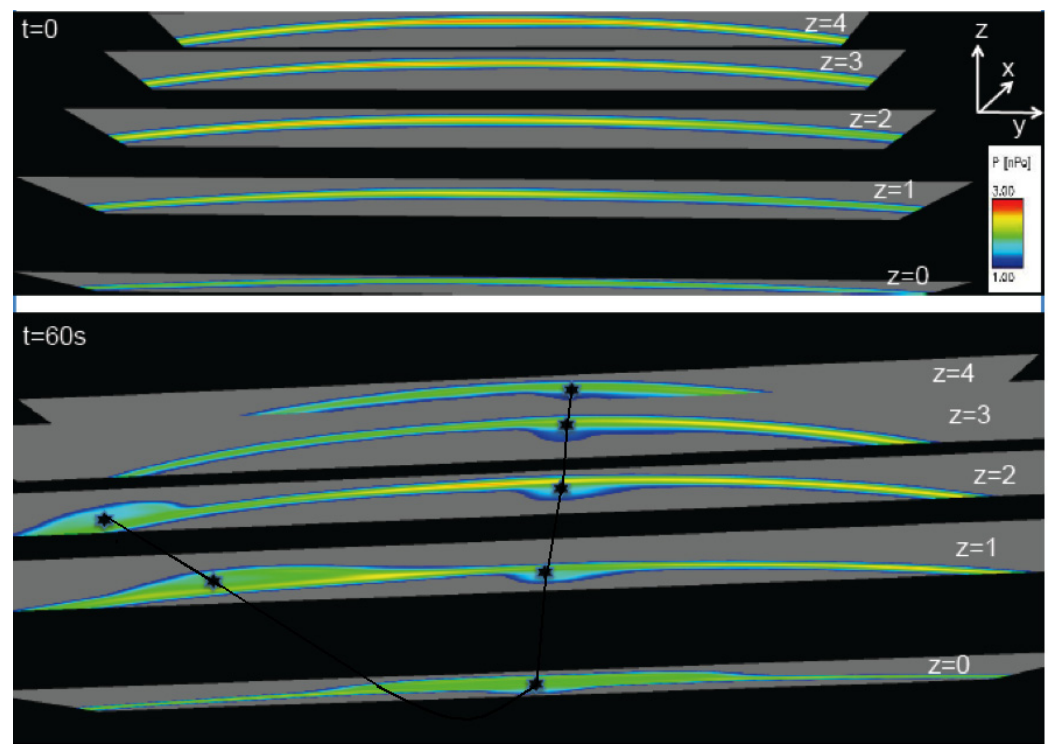

Fig. 10. Plasma pressure evolution for the $z$ cuts $\left(z=0,1,2,3,4 R_{\mathrm{E}}\right)$ at $t=0$ (top image) and $t=60 \mathrm{~s}$ (bottom image). The observer sees the plots from bottom to top and anti-sunward.

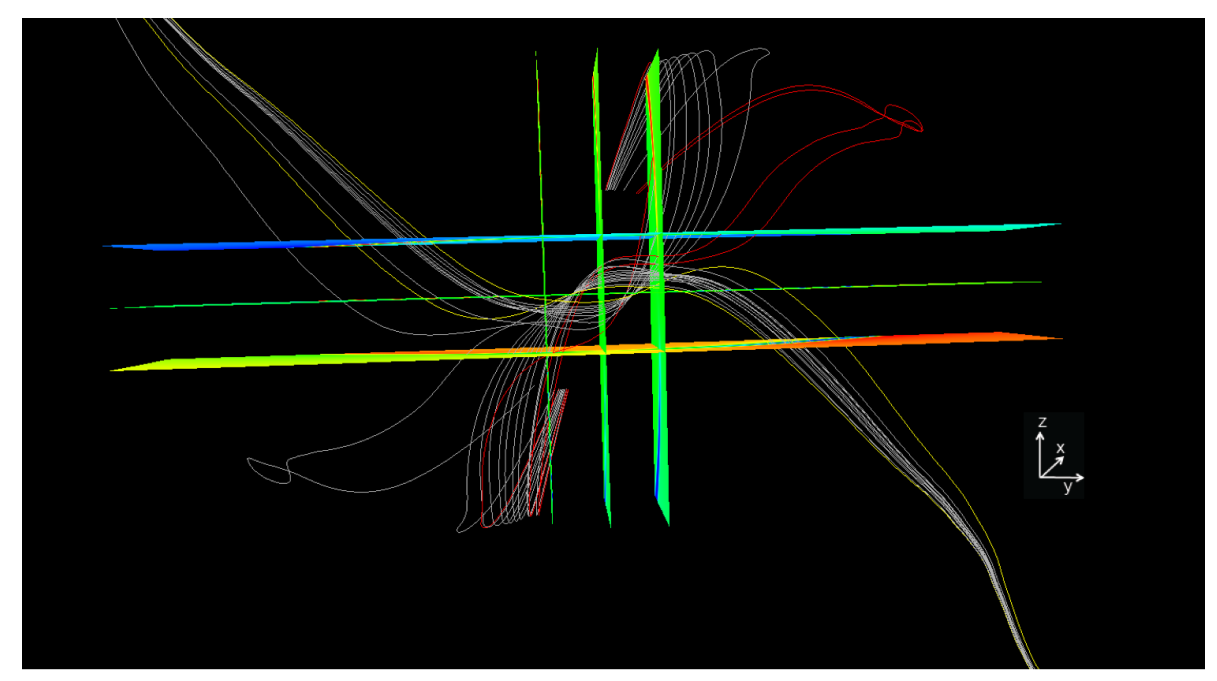

Fig. 11. Reconnected magnetic field lines drawn at $t=30 \mathrm{~s}$. The lines exhibit the interlinked flux tubes configuration.

at $y>0$ (duskward, right side), but bulges inward into the magnetosphere near $y=0$. We observed that the same flux tube has parts bulging into the magnetosphere and other parts bulging into the magnetosheath. Ku and Sibeck (2000) obtained bulges in their MHD simulation followed by islands. The bulges were confined to the magnetosheath while the islands generated significant signatures in the magnetosphere.

The magnetic field lines at $t=30 \mathrm{~s}$ in Fig. 11 show that the two elbow-shaped flux tubes are interconnected at the subsolar magnetopause. The interlinked tubes are composed of lines connected to the northern on the south side and to the southern on the north side, as indicated in Fig. 4b. These field lines seem to be pulling against each other, forming a blob at the subsolar region.

The flow velocity in the $y$ direction $\left(v_{y}\right)$ confirms our previous statement about the peculiar configuration of the interlinked flux tubes. Figure 12 shows $v_{y}$ on the $x-y$ plane for several $z$ cuts $(z=-2,-1,0,1,2 R \mathrm{E})$ seen from two different perspectives: from above (top) and below (bottom). The lines (blue and red) have been drawn to indicate the shape of the flux tubes. One flux tube is clearly identifiable as being located in the Northern Hemisphere, trying to move dawnward (blue spots), while the other flux tube in the Southern Hemisphere is trying to move duskward (red spots). 

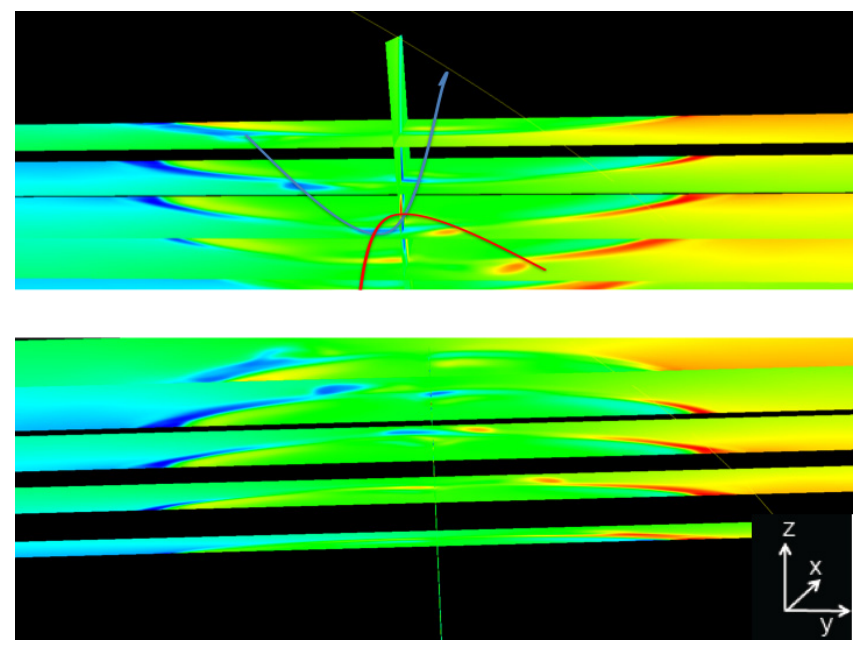

Fig. 12. Flow velocity in the $y$ direction $\left(v_{y}\right)$ for $z$ cuts $(z=-2$, $-1,0,1,2 R_{\mathrm{E}}$ ) seen from two different perspectives: from above (top) and from below (bottom). Blue and red mean roughly dawnward and duskward flow, respectively. Red and blue lines have been drawn to indicate the flux tube shape.

Our simulation results have shown an FTE formed by the two elbow-shaped flux tubes interconnected at the subsolar region. This special FTE seems to be generated by multiple $X$ lines (sketch in Fig. 4). An important question is if the multiple $X$ lines could be observed far from the subsolar region. Also, it is important to understand what happened to the extended $X$ line seen before the onset of the interlinked flux tubes. Let us then analyze the $X$ line behavior at $t=0$, the event onset. Figure 13 shows the magnetic topology at $t=0$ for positive $z$ cuts at $z=0.6$ and $1 R_{\mathrm{E}}$ (first and middle plots, respectively). The reconnection points are indicated by X's. The third plot is just a zoom of the $X$ point region at $z=1 R_{\mathrm{E}}$ (middle plot). We have also checked the negative $z$ cuts at $z=-0.6$ and $-1 R_{\mathrm{E}}$. The $X$ points in the Southern Hemisphere are located at the dawn side (negative $y$ ), as a mirror of Fig. 13. The single and extended $X$ line observed below $z=-0.6 R_{\mathrm{E}}$ and above $z=0.6 R_{\mathrm{E}}$ is tilted from southerndawn to northern-dusk.

On the other hand, when we looked closer at the subsolar region (between $z=-0.6$ and $0.6 R_{\mathrm{E}}$ ), we suggested the existence of the three $X$ points indicated in the middle sketch of Fig. 4a. We examined the magnetic topology at $t=0$ for other planes but than the noon-midnight (middle plot of Fig. 9) in order to picture the event onset more accurately in 3-D.

We investigated the magnetic topology in the Southern and Northern Hemispheres at the event onset $t=0$. Figure 14 shows the magnetic topology for the equatorial plane $(z=0)$ and for the Northern Hemisphere at $z=0.2$ and $0.43 R_{\mathrm{E}}$. The right panel in Fig. 14 shows a symmetric picture of the reconnected magnetic field, indicated by the green and yellow colors. The green bulges into the magnetosheath from $y=0$

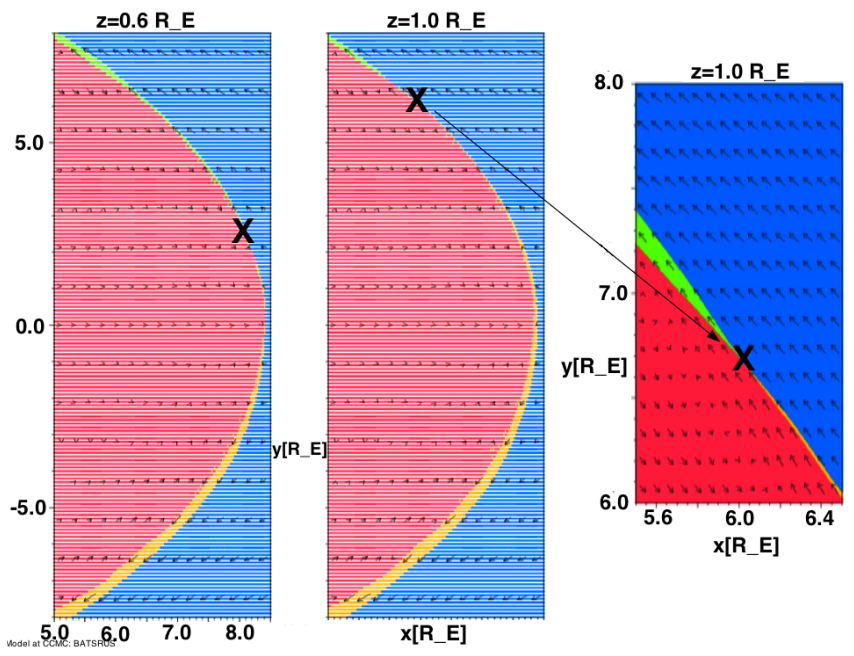

Fig. 13. Magnetic topology at $t=0$ for positive $\mathrm{z}$ cuts at $z=0.6$ and $1 R_{\mathrm{E}}$ (first and middle plots), showing the $X$ points. The third plot shows a zoom of the $X$ point region at $z=1 R_{\mathrm{E}}$. Colors are related to magnetic topology as red, closed; yellow, connected to north; green, connected to south; and blue, solar wind. Arrows indicate flow velocity.
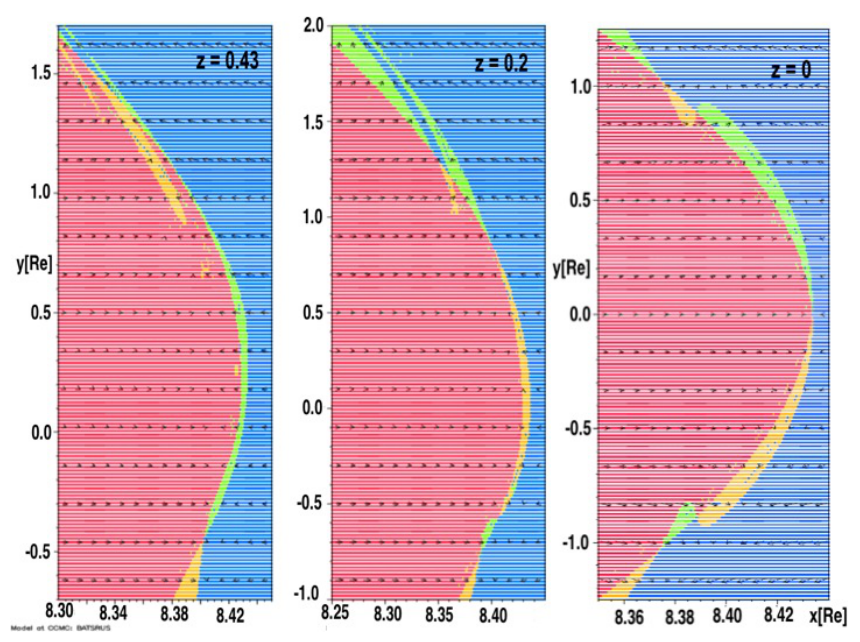

Fig. 14. Magnetic topology at $t=0$ for $z=0.43, z=0.2$ and $z=$ $0 R_{\mathrm{E}}$. Colors are related to magnetic topology as red, closed; yellow, connected to north; green, connected to south; and blue, solar wind. Arrows indicate flow velocity.

to $0.9 R_{\mathrm{E}}$ and the yellow from $y=-0.9$ to $0 R_{\mathrm{E}}$, while the green at $y=0.8$ to $1 R_{\mathrm{E}}$ and the yellow at $y=-1$ to -0.8 bulges into the magnetosphere. There is a yellow blob at $z=0.2 R_{\mathrm{E}}$ when looking at the noon-midnight plane shown in the middle plot of Fig. 9. The yellow blob is located from $z=0$ to $z=0.35 R_{\mathrm{E}}$. Its extent is about $0.35 R_{\mathrm{E}}$ in the $z$ direction. This flux tube is the one that bulges outward into the magnetosheath from $y=-0.9$ to $0 R_{\mathrm{E}}$ observed in the right panel of Fig. 14. At $z=0.43 R_{\mathrm{E}}$, there is a green blob located between $z=0.35$ to $0.55 R_{\mathrm{E}}$ for the noon-midnight plane in 


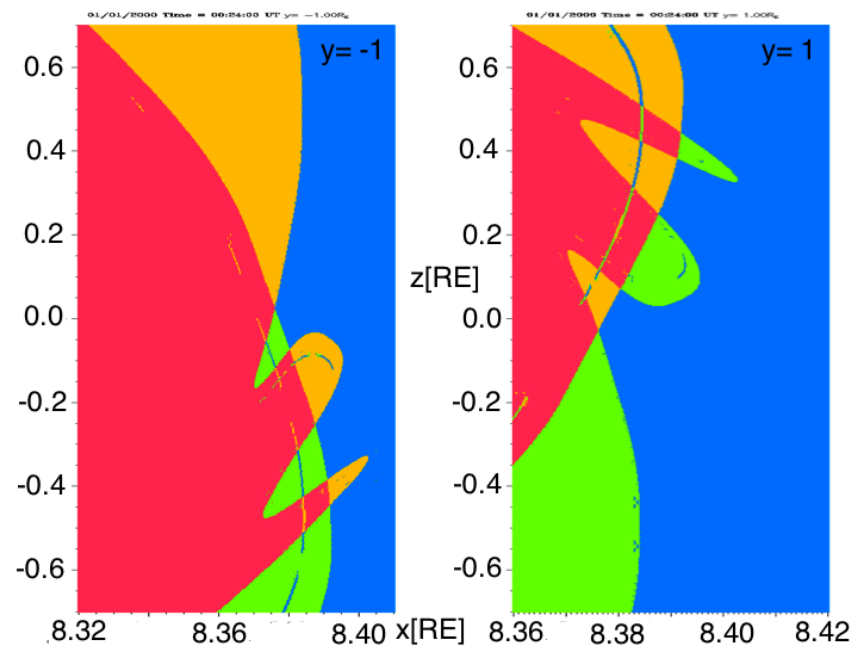

Fig. 15. Magnetic topology at $t=0$ for $y=-1$ and $1 R_{\mathrm{E}}$. Colors are related to magnetic topology as red, closed; yellow, connected to north; green, connected to south; and blue, solar wind.

the middle panel of Fig. 9. This green blob is stretched from $y=-0.5$ to $1.5 R_{\mathrm{E}}$ for the $z=0.43 R_{\mathrm{E}}$ plane displayed in the left panel in Fig. 14. We have also checked the $z$ cuts in the Southern Hemisphere for $z=-0.2$ and $-0.43 R_{\mathrm{E}}$. These plots are just a reverse of Fig. 14 and we do not show them here.

Also at $t=0$, we analyzed the magnetic topology for the dawn and dusk sides. Figure 15 displays the magnetic topology for $y=-1$ (left panel) and $1 R_{\mathrm{E}}$ (right panel). At the dawn side $(y=-1)$, the green and yellow blobs are located in the Southern Hemisphere. The green and yellow blobs lie in the magnetosphere and in the magnetosheath, respectively. This configuration indicates that the tubes connected to the southern (green) may be attached directly to Earth and the tubes connected to the northern (yellow) may be open to the IMF. The opposite is observed for the dusk side $(y=1)$. The blobs are located mostly in the Northern Hemisphere. The yellow blobs are located in the magnetosphere and the green ones in the magnetosheath. The tubes connected to the northern (yellow) may be now open to the IMF and the tubes connected to the southern (green) may be attached directly to Earth. The tubes open to the IMF seem to be pulled by the tubes connected to Earth. The tubes between $y=-1$ to $1 R_{\mathrm{E}}$ are oriented in the same direction as the extended $X$ line observed at the first stage of the simulation. We did not observe the elbow shape in the tubes at the event onset $(t=0)$.

Figures 9,14 and 15 suggest the existence of four interlinked flux tubes at $t=0$. These structures of smaller scale evolve to the final structure of only a set of two interconnected flux tubes of larger scale (Fig. 4b).

We identified two regions with different characteristics at $t=0$ : (1) between $z=-0.6$ and $0.6 R_{\mathrm{E}}$; and (2) below $z=-0.6 R_{\mathrm{E}}$ and above $z=0.6 R_{\mathrm{E}}$. The $X$ line configuration for the subsolar region, between $z=-0.6$ and $0.6 R_{\mathrm{E}}$,
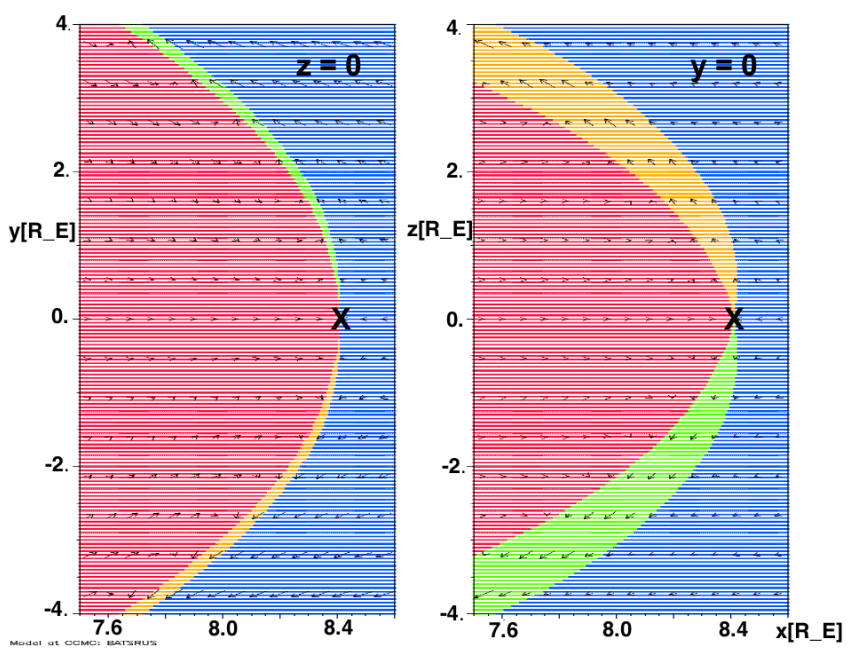

Fig. 16. Magnetic topology at $1 \mathrm{~min}(t=-1 \mathrm{~min})$ before the interlinked flux tubes onset for the noon-midnight plane $(z=0$, first plot) and for the equatorial plane ( $y=0$, second plot). The $X$ point is coincident with the subsolar point. Colors are related to magnetic topology as red, closed; yellow, connected to north; green, connected to south; and blue, solar wind. Arrows indicate flow velocity.

is composed of multiple reconnection lines (Lee and Fu, 1985). For the Northern and Southern Hemispheres, below $z=-0.6 R_{\mathrm{E}}$ and above $z=0.6 R_{\mathrm{E}}$, we observed only a single and extended line oriented from southern dawn to northern dusk

Apparently two reconnection modes seem to be occurring simultaneously in different locations at $t=0$ : (1) the multiple $X$ lines (three) on a localized region showing a timedependent, patchy and multiple reconnection for the subsolar region (between $z=-0.6$ and $0.6 R_{\mathrm{E}}$ ); and (2) the single and extended $X$ line indicating a steady and large-scale reconnection for the region beyond the subsolar site (below $z=-0.6 R_{\mathrm{E}}$ and above $\left.z=0.6 R_{\mathrm{E}}\right)$.

The magnetic reconnection features establish the extent of an FTE (Fear et al., 2008). The patchy reconnection is active only on a localized region and this may work as a limit on the FTE extent (Russell and Elphic, 1978). Thus the interlinked flux tubes were apparently limited to the patchy reconnection region.

\subsection{A glance after the interlinked flux tubes event}

The two interlinked flux tubes grew at the subsolar region over a time period of about four minutes. After that, the two tubes moved northward as a single structure. They moved away from the reconnection site due to the magnetic tension and the magnetosheath flow (Lee et al., 1993; Fear et al., 2008).

The interlinked tubes event modified the $X$ point configuration on the magnetopause. Before the event, the $X$ point was coincident with the subsolar point. Figure 16 shows the 

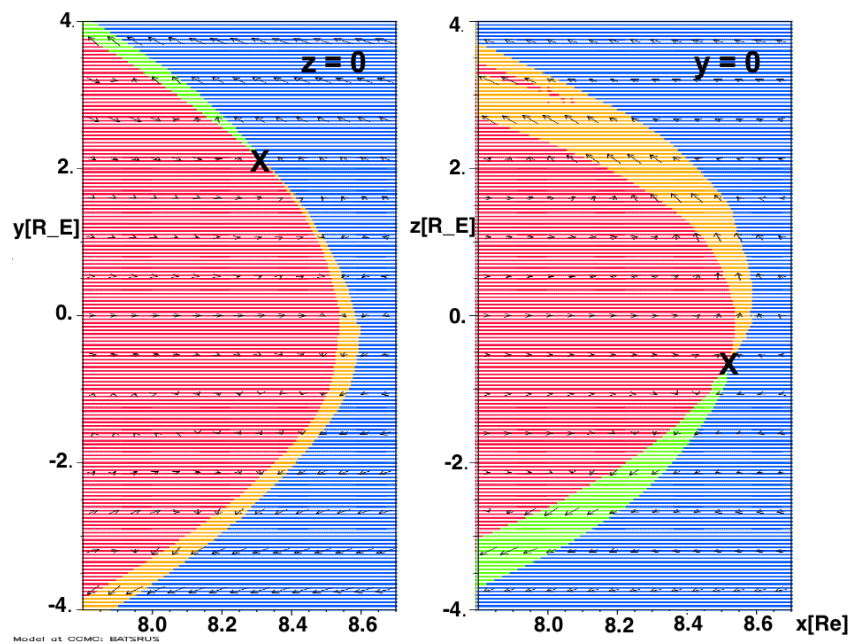

Fig. 17. Magnetic topology at $t=6 \mathrm{~min} 30 \mathrm{~s}$ for the noon-midnight plane $(z=0$, first plot) and for the equatorial plane $(y=0$, second plot). The $X$ point is shifted from the subsolar point and located at $z=-0.7$ and $y=2.2 R_{\mathrm{E}}$. Colors are related to magnetic topology as red, closed; yellow, connected to north; green, connected to south; and blue, solar wind. Arrows indicate flow velocity.

magnetic topology for the noon-midnight plane $(z=0$, first plot) and for the equatorial plane $(y=0$, second plot) before the event onset at $t=-1 \min (1 \mathrm{~min}$ before $t=0)$. The $X \mathrm{~s}$ in Fig. 16 show the subsolar $X$ point. When the interlinked tubes had been completely formed we observed two $X$ points. The right panel in Fig. 4a illustrates the two $X$ points during the main event. The two interconnected flux tubes move collectively northward and drag the northern $X$ point. The remaining single $X$ point is the one located in the south and dusk side ( $y=2.2$ and $\left.z=-0.7 R_{\mathrm{E}}\right)$ at $t=6 \mathrm{~min}$ $30 \mathrm{~s}$ (Fig. 17). The interlinked tubes event (formation and propagation) shifted the $X$ point from the subsolar site (configuration before the event in Fig. 16) to the Southern dusk Hemisphere (configuration after the event in Fig. 17).

After the interlinked flux tubes had propagated away and the remaining $X$ point had found a new location, we observed new magnetic structures. Figure 18 shows the magnetic topology at $t=7 \mathrm{~min}(7 \mathrm{~min}$ after $t=0)$ for the noonmidnight plane $(y=0)$. The first plot shows the numbered magnetic structures $(1,2,3)$ that are displayed in the other three plots. The structures 2 and 3 indicate two flux tubes formed by lines connected to the Northern and Southern Hemispheres, respectively. The structure 2 is bulging outward into the magnetosheath and the structure 3 is bulging inward into the magnetosphere at $y=0$. The tube in panel 2 moves northward while the structure 3 propagates southward. The structure 1 is formed by two blobs. The yellow blob on the bottom is formed by reconnected lines linked to the Northern Hemisphere. The green blob on the top is composed of semi-open lines connected to Southern Hemisphere. The blobs are not symmetric at $y=0$. The blobs

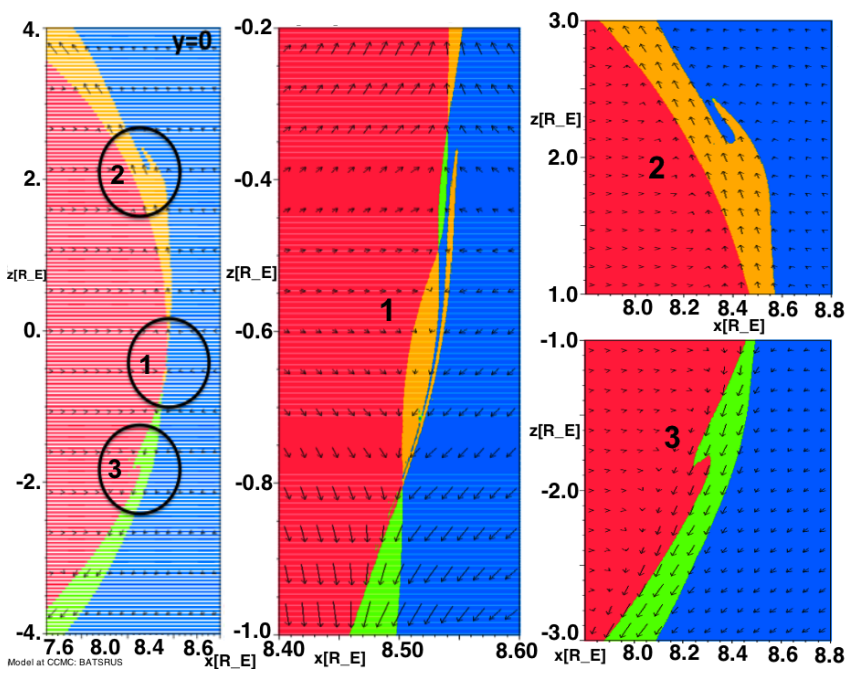

Fig. 18. Magnetic topology after the interlinked flux tubes had propagated northward, at $t=7 \mathrm{~min}$, for the noon-midnight plane ( $y=0$, first plot), showing the numbered magnetic structures $(1,2,3)$ displayed on a larger scale on the other three plots. Colors are related to magnetic topology as red, closed; yellow, connected to north; green, connected to south; and blue, solar wind. Arrows indicate flow velocity.

seem to be similar to the interlinked flux tubes, although on a smaller scale.

It is important to notice that the interlinked flux tubes event described in Sect. 3.1 was not the only peculiar magnetic topology pattern found in our simulation. A similar structure occurred later, as shown in Fig. 18. Also, the large-scale interlinked flux tubes (Sect. 3.1) appear to be a magnetic topology evolution of small-scale interlinked tubes.

\section{Conclusions}

This paper presents results from a global 3-D MHD simulation under a large south-duskward IMF. Only one run has been examined in detail. We have obtained several FTE-like structures during $45 \mathrm{~min}$ of simulation, in spite of the solar wind conditions being kept constant (Raeder, 2006; Dorelli and Bhattacharjee, 2009).

Our main finding is a large-scale magnetic structure of two interlinked elbow-shaped flux tubes (Fig. 4b) at the subsolar region of the dayside magnetopause after about $24 \mathrm{~min}$ of simulation. We found other interlinked tubes in our simulation. The magnetic topology of the interconnected tubes identified in our study seems to be similar in nature, although with different spatial scales. Small-scale interlinked tubes were found before and after the main event. Two sets of small-scale interlinked flux tubes developed into the largescale interlinked tubes, the main event in this paper. After the main event had propagated away, we observed a set of two small-scale flux tubes. 
The low resistivity in our simulation allowed the interlinked flux tubes to form and grow. We used a high grid resolution at the magnetopause region. This may have suppressed the numerical diffusion in our simulation. Raeder (2006) pointed out that FTEs do not develop if the resistivity is high (low resolution). Even if the FTEs can be formed they will propagate away quickly. The recent improvement of grid resolution has been explained by Raeder (2006) as the main reason that FTEs can now be reproduced in simulations. We can extend that to explain our capability to obtain the interlinked flux tubes.

We investigated if the large-scale interconnected tubes exhibit FTE features (Russell and Elphic, 1978; Haerendel et al., 1978). The tubes did not present clear FTE signatures independently. However, the set of the two tubes showed the main characteristics of bipolarity of the normal component magnetic field and the core enhancement of the magnetic field magnitude. The two tubes altogether were identified as a unique FTE. We did not observe a twisting of the magnetic field lines in agreement with the interlinked flux tubes obtained by Hesse et al. (1990). However, Russell and Elphic (1978) identified such twisting of the magnetic lines in their FTEs.

Our FTE is formed by two flux tubes (Fig. 4b). Each tube has the "elbow" configuration that is similar to the model of Russell and Elphic (1978). The two interconnected tubes have one side connected to Earth and the other to the IMF. At the subsolar region the tube on the top is connected to the Southern Hemisphere and the tube on the bottom to the northern side. The tube on the top bulges outward into the magnetosheath at $y<0$ (dawnward, left side), but inward into the magnetosphere near $y=0$ (Fig. 10). Symmetrically the bottom tube bulges outward into the magnetosheath at $y>0$ (duskward, right side), but inward into the magnetosphere near $y=0$.

We investigated the magnetic topology and described the evolution of the $X$ line configurations during the interlinked tubes event (Fig. 4a). Previous to the interlinked tubes onset, a single and extended $X$ line was observed to run from southern dawn to northern dusk for the south-duskward IMF, in agreement with Gonzalez and Mozer (1974). At the interlinked event onset $(t=0)$, the magnetic topology changed and two new $X$ points appeared in the north and south sides. The new southern and northern $X$ points joined to the subsolar reconnection point, composing the three $X$ point configuration. The evolution of this configuration showed that the subsolar $X$ point vanished and the reconnection was suppressed. Only the more recently formed two southern and northern $X$ points remained at the magnetopause. These two $X$ lines generated the interlinked tubes. In summary, the scenario changed from a single to multiple reconnection lines.

Nevertheless, the evolution of the $X$ point configurations described previously did not occur throughout the entire magnetopause, but instead in a localized region. At the interlinked flux tubes onset $(t=0)$, two reconnection modes occurred simultaneously near and far from the subsolar region. We observed localized multiple lines at the subsolar region (between $z=-0.6$ and $0.6 R_{\mathrm{E}}$ ), and only a single and extended line away from the subsolar spot (below $z=$ $-0.6 R_{\mathrm{E}}$ and above $z=0.6 R_{\mathrm{E}}$ ). This single $X$ line was oriented from southern dawn to northern dusk, maintaining the configuration previous to the interlinked tubes event. In summary, the two simultaneous reconnection modes observed at the interlinked flux tubes onset $(t=0)$ were (1) the multiple $X$ lines (three) on a localized region, showing a timedependent, patchy and multiple reconnection for the subsolar region (between $z=-0.6$ and $0.6 R_{\mathrm{E}}$ ); and (2) the single and extended $X$ line, indicating a steady and large-scale reconnection for the region beyond the subsolar site (below $z=-0.6 R_{\mathrm{E}}$ and above $z=0.6 R_{\mathrm{E}}$ ).

We observed that the responsible process for the interconnected tubes generation was the time-dependent, patchy and multiple reconnection. Our results are in agreement with Lee et al. (1993) who attributed the generation of the interlinked tubes to a combination of multiple $X$ lines and patchy reconnection. Otto (1995) also pointed out that reconnection of at least two patches would produce the interconnected tubes, but the process would be more likely to occur if multiple patches existed (Nishida, 1989). In the FTEs scenario, our results are a combination of patchy and time-dependent reconnection proposed by Russell and Elphic (1978), and the multiple $X$ lines found by Lee and Fu (1985).

The two interlinked flux tubes grew at the subsolar region over a time period of about four minutes. Before the event, the $X$ point was coincident with the subsolar point. When the interlinked tubes had been completely formed, we observed two $X$ points at the Northern and Southern Hemispheres. The two interconnected flux tubes moved collectively northward and dragged the northern $X$ point. The remaining single $X$ point was the one located in the south and dusk side. The interlinked tubes event (formation and propagation) shifted the $X$ point from the subsolar site (Fig. 16) to the Southern dusk Hemisphere (Fig. 17). The new flux tubes were formed at locations shifted from the subsolar point and did not reach large sizes like the main event.

We point out some observable characteristics of the interlinked tubes that may be helpful on a spacecraft data search. Moving from the magnetosphere to the magnetosheath, $B_{x}$ (approximated as the normal component of the magnetic field $B_{\mathrm{n}}$ ) increases positively in the northern side and becomes more negative in the Southern Hemisphere. The magnetic field magnitude $B$ presents a decrease in both tubes when the satellites get closer to the them in the magnetospheric side. The magnitude $B$ decreases again when the tubes start growing. The tubes grow into the magnetosheath and the system becomes anti-symmetric in the $x$ direction. When the tubes start propagating northward, $B$ increases in the northern side while it decreases in the Southern Hemisphere. $B_{z}$ decreases from the magnetosphere to the magnetosheath, as we expect. If the spacecraft moves from the Southern to the 
Northern Hemisphere, $B_{x}$ shows the symmetric bipolar characteristic and the magnetic field magnitude $B$ presents the core enhancement. Our magnetic topology study may also help when examining multi-spacecraft data. The sense of streaming particles in the events describes the connection to magnetosphere.

We analyzed one run in this work and therefore the generality of our results remains unknown. However, we expect them to be typical because we chose typical solar wind values. The interlinked elbow-shaped flux tubes have been previously obtained in several models and data (Hesse et al., 1990; Lee et al., 1993; Otto, 1995; Louarn et al., 2004). This structure has been usually modeled under the conditions of high grid resolution, negative $B_{z}$ and finite $B_{y}$. The interlinked tubes may be a common feature of magnetopause reconnection (Hesse et al., 1990; Otto, 1995; Louarn et al., 2004). Nishida (1989) and Otto (1995) showed that reconnection at multiple points would produce many sets of interconnected tubes at the magnetopause. The reconnection process occurring at many different size patches associated with different reconnection rates would produce a mixture of open and closed magnetic flux tubes. Adding to this, we should still consider the reconnection at the interface of the interlinked tubes. This complex scenario could explain the lowlatitude boundary layer (LLBL) existence (Nishida, 1989; Otto, 1995). Louarn et al. (2004) observed the structure of interlinked flux tubes in Cluster data and explained that the complex geometrical properties of FTEs could be related to the interlinked topology created by the motion of the reconnected flux tubes.

In our simulation, the scenario for the interlinked tubes development was highly symmetric in relation to the Sun-Earth line. We did not consider dipole tilt. Otto (1995) obtained the interconnected tubes in MHD simulation but neglected dipole tilt as well. Raeder (2006) discussed the role of the dipole tilt in his MHD simulations. His results showed that dipole tilt is required for FTEs formation during southward IMF. In our simulation we considered a large $B_{y}$ component added to a southward IMF and we were able to obtain the FTEs. Adding dipole tilt to our setting would be an interesting topic for a future work. Under these conditions we are not sure if the interlinked tubes could be formed since the flow could pinch the magnetopause and easily generate new $X$ points. If they could be formed they would probably propagate away quickly with the magnetosheath flow. The dipole tilt would probably change all the dynamics in our simulation, as it did in Raeder's (2006).

Acknowledgements. This work was carried out using the SWMF/BATSRUS tool developed at the University of Michigan Center for Space Environment Modeling (CSEM) and made available through the NASA Community Coordinated Modeling Center (CCMC). We gratefully acknowledge Fapesp - Fundação de Amparo à Pesquisa do Estado de São Paulo (2010/10934-2), CNPq - Conselho Nacional de Desenvolvimento Científico e Tecnológico
(160131/2012-0), CCMC at Goddard Space Flight Center (GSFC) (http://ccmc.gsfc.nasa.gov), the BATS-R-US developers at the CSEM, and the visualization tool SWX developers at NASA GSFC. We also thank the referees for their valuable contribution to our paper.

Topical Editor L. Blomberg thanks J. De Keyser and one anonymous referee for their help in evaluating this paper.

\section{References}

Dorelli, J. C. and Bhattacharjee, A.: On the generation and topology of flux transfer events, J. Geophys. Re., 114, A06213, doi:10.1029/2008JA013410, 2009.

Fear, R. C., Milan, S. E., Fazakerley, A. N., Lucek, E. A., Cowley, S. W. H., and Dandouras, I.: The azimuthal extent of three flux transfer events, Ann. Geophys., 26, 2353-2369, doi:10.5194/angeo-26-2353-2008, 2008.

Fedder, J., Slinker, S., Lyon, J., and Russel, C. T.: Flux transfer events in global numerical simulations of the magnetosphere, J. Geophys. Res., A5, 1048, doi:10.1029/2001JA000025, 2002.

Gombosi, T., Powell, K., Zeeuw, D. D., Clauer, C., Hansen, K., Manchester, W., Ridley, A., Roussev, I., Sokolov, I., Stout, Q., and Tóth, G.: Solution Adaptive MHD for Space Plasmas: Sunto-Earth Simulations, Comp. Sci. Eng., 6, 14-35, 2004.

Gonzalez, W. and Mozer, F.: A quantitative model for the potential resulting from reconnection with an arbitrary interplanetary magnetic field, J. Geophys. Res., 79, 4186-4194, 1974.

Haerendel, G., Paschmann, G., Sckopke, N., Rosenbauer, H., and Hedgecock, P.: The frontside boundary layer of the magnetosphere and the problem of reconnection, J. Geophys. Res., 83, 3195-3216, 1978.

Hesse, M., Birn, J., and Schindler, K.: On the topology of flux transfer events, J. Geophys. Res., 95, 6549-6560, 1990.

Ku, H. C. and Sibeck, D. G.: Flux transfer events produced by bursty merging at a single $X$ line, J. Geophys. Res., 103, 14965-14978, 1998.

Ku, H. C. and Sibeck, D. G.: Flux transfer events produced by the onset of merging at multiple $X$ lines, J. Geophys. Res., 105, 2657-2675, 2000.

Kuznetsova, M., Hesse, M., Rastaetter, L., Taktakishvili, A., Toth, G., Zeeuw, D. L. D., Ridley, A., and Gombosi, T.: Multiscale modeling of magnetospheric reconnection, J. Geophys. Res., 112, A10210, doi:10.1029/2007JA012316, 2007.

Kuznetsova, M., Sibeck, D., Hesse, M., Wang, Y., Rastaetter, L., Toth, G., and Ridley, A.: Cavities of weak magnetic field strength in the wake of FTEs: Results from global magnetospheric MHD simulations, Geophy. Res. Lett., 36, L10104, doi:10.1029/2009GL037489, 2009.

Lee, L. C. and Fu, Z. F.: A theory of magnetic flux transfer at the Earth's magnetpause, Geophys. Res. Lett., 12, 105-108, 1985.

Lee, L. C., Ma, Z., and Otto, A.: Topology of magnetic flux ropes and formation of fossil flux transfer events and boundary layer plasmas, J. Geophys. Res., 98, 3943-3951, 1993.

Louarn, P., Federov, A., Budnik, E., Fruit, G., Sauvaud, J. A., Harvey, C. C., and Dandouras, I.: Cluster observations of complex 3D magnetic structures at the magnetopause, Geophys. Res. Lett., 31, L19805, doi:10.1029/2004GL020625, 2004. 
Moen, J. and Brekke, A.: The solar flux infuence of quiet-time conductances in the auroral ionosphere, Geophys. Res. Lett., 20, 971-874, doi:10.1029/92GL02109, 1993.

Nishida, A.: Can random reconnection on the magnetopause produce the low latitude boundary layer, Geophys. Res. Lett., 16, 227-230, 1989.

Ogino, T., Walker, R. J., and Ashour-Abdalla, M.: A magnetohydrodynamic simulation of the formation of magnetic flux tubes at the Earth's dayside magnetopause, Geophys. Res. Lett., 16, 155-158, 1989.

Otto, A.: Forced three-dimensional magnetic reconnection due to linkage of magnetic flux tubes, J. Geophys. Res., 100, 1186311874, 1995.

Paschmann, G., Haerendel, G., Papamastorakis, I., Sckopke, N., Bame, S., Gosling, J., and Russell, C.: Plasma and magnetic field characteristics of Magnetic Flux Transfer Events, J. Geophys. Res., 87, 2159-2168, 1982.

Powell, K., Roe, P., Linde, T., Gombosi, T., and Zeeuw, D. D.: A solution adaptative upwind scheme for ideal magnetohydrodynamics, J. Comput. Phys., 154, 284-309, 1999.

Raeder, J.: Flux Transfer Events: 1. generation mechanism for strong southward IMF, Ann. Geophys., 24, 381-392, doi:10.5194/angeo-24-381-2006, 2006.

Ridley, A. J., Gombosi, T. I., and DeZeeuw, D. L.: Ionospheric control of the magnetosphere: conductance, Ann. Geophys., 22, 567-584, doi:10.5194/angeo-22-567-2004, 2004.
Rijnbeek, R. and Cowley, S.: Magnetospheric flux erosion events are flux transfer events, Nature, 309, 135-138, 1984.

Robinson, R., Vondrak, R. R., Miller, K., Dabbs, T., and Hardy, D. A.: On calculating ionospheric conductances from the flux and energy of precipitating electrons, J. Geophys. Res., 92, 25652569, doi:10.1029/JA092iA03p02565, 1987.

Russell, C. T. and Elphic, R. C.: Initial ISEE Magnetometer Results: Magnetopause observations, Space Sci. Rev., 22, 681-715, 1978.

Scholer, M.: Strong core magnetic fields in magnetopause flux transfer events, Geophys. Res. Lett., 15, 748-751, 1988.

Sibeck, D., Kuznetsova, M., Angelopoulos, V., Glaßmeier, K.H., and McFadden, J.: Crater FTEs: simulation results and THEMIS observations, Geophys. Res. Lett., 35, L17S06, doi:10.1029/2008GL0335682008.

Southwood, D., Farrugia, C., and Saunders, M.: What are flux transfer events, Planet. Space Sci., 36, 503-508, 1988.

Toth, G., Sokolov, I. V., Gombosi, T. I., Chesney, D. R., Clauer, C. R., De Zeeuw, D. L., Hansen, K. C., Kane, K. J., Manchester, W. B., Oehmke, R. C., Powell, K. G., Ridley, A. J., Roussev, I. I., Stout, Q. F. and Volberg, O., Wold, R. A., Sazykin, S., Chan, A., Yu, B., and Kóta, J.: Space weather modeling framework: a new tool for the space science community, J. Geophys. Res., 110, A12226, doi:10.1029/2005JA011126, 2005.

Treumann, R. A. and Baumjohann, W.: Advanced Space Plasma Physics, Imperial College Press, London, 1997. 